\title{
CORRIGENDUM
}

\section{Peripheral blood mononuclear cell gene expression profiles identify emergent post-traumatic stress disorder among trauma survivors}

Molecular Psychiatry (2005) 10, 514.

doi:10.1038/sj.mp.4001654

Correction to: Molecular Psychiatry 10, 500-513. doi:10.1038/sj.mp.4001636

Following the 1 February 2005 advance online publication of this article, the authors noted the following error:
In the Supplementary Notes of this article, the password and username of the GEO deposit were included by mistake. The only information required to access the data are the GEO serial numbers provided. The username and password are not required. 